\title{
High STOP-BANG questionnaire scores predict intraoperative and early postoperative adverse events
}

Edwin $\underline{\text { Seet }}^{1}$, MBBs, MMed, Maureen $\underline{\text { Chua }}^{1}$, MBBs, MMed, Chen Mei Liaw ${ }^{1}$, MBBs, MMed

INTRODUCTION Obstructive sleep apnoea (OSA) is the most common sleep-related breathing disorder associated with multisystemic organ involvement. The STOP-BANG questionnaire is a concise, validated questionnaire that is used to screen for OSA. This study aimed to establish the use of the STOP-BANG questionnaire for perioperative patient risk stratification.

METHODS In this retrospective cohort study, we extracted the demographic, medical and perioperative outcome data of all patients who underwent elective surgery, excluding ophthalmic surgeries, from January to December 2011. Multivariate regression analysis was used to predict independent risk factors for intraoperative and early postoperative adverse events. RESULTS Of the 5,432 patients analysed, $7.4 \%$ had unexpected intraoperative and early postoperative adverse events. We found that the risk of unexpected intraoperative and early postoperative adverse events was greater in patients with STOP-BANG scores $\geq 3$ compared to those with a STOP-BANG score of 0 (score 3: odds ratio [OR] 3.6, 95\% confidence interval [CI] 2.1-6.3, p < 0.001; score 4: OR 3.4, 95\% Cl 1.8-6.5, p<0.001; score 5: OR 6.4, 95\% Cl 2.7-15.0, p<0.001; score $\geq 6$ : OR 5.6, 95\% Cl 2.1-15.4, p < 0.001). Patients with STOP-BANG scores $\geq 5$ had a fivefold increased risk of unexpected intraoperative and early postoperative adverse events, while patients with STOP-BANG scores $\geq 3$ had a 'one in four' chance of having an adverse event. Other independent predictors included older age $(p<0.001)$, American Society of Anesthesiologists class $\geq 2(p<0.003)$ and uncontrolled hypertension $(p=0.028)$.

CONCLUSION STOP-BANG score may be used as a preoperative risk stratification tool to predict the risk of intraoperative and early postoperative adverse events.

Keywords: adverse events, obstructive sleep apnoea, perioperative complications, STOP-BANG questionnaire

\section{INTRODUCTION}

Obstructive sleep apnoea (OSA) is the most common sleep-related breathing disorder. It is a clinical syndrome that is characterised by recurrent, episodic cessation of breathing that lasts for ten seconds or more during sleep. The prevalence of OSA is estimated to be $41.5 \%$ among patients presenting for elective surgery. ${ }^{(1)}$ This number increases up to $80 \%$ in high-risk patients such as those who are scheduled to receive bariatric surgery. ${ }^{(2)}$

OSA remains undiagnosed in about four out of five patients at the time of surgery; $;^{(2)}$ this is likely due to logistical difficulties and cost considerations in diagnosing patients via a formal polysomnography study. As OSA has been associated with increased postoperative cardiac complications, respiratory failure, emergent reintubations and postoperative intensive care unit admissions, ${ }^{(3)}$ patients with undiagnosed OSA may unknowingly be placed at an increased risk for perioperative complications, due to the untreated nature of their disease. Furthermore, OSA poses an economic burden, as it increases the length of hospital stay and healthcare costs. ${ }^{(4)}$ As such, various clinical prediction scores have been employed to screen for OSA preoperatively.

The STOP-BANG questionnaire (Table I) is an 8-point scoring system that is routinely administered during preoperative assessment to screen for OSA. ${ }^{(5)}$ The acronym STOP-BANG stands for Snoring loudly, Tiredness in daytime, Observed apnoea during sleep, high blood Pressure, Body mass index $>35 \mathrm{~kg} / \mathrm{m}^{2}$, Age $>50$ years, Neck circumference $>40 \mathrm{~cm}$, and male Gender.
STOP-BANG scores range from 0 to 8 ; a score $\geq 3$ means that the patient is at risk of OSA, while a score $\geq 5$ indicates that the patient is at high risk of OSA. ${ }^{(6)}$ The STOP-BANG questionnaire has been shown to have a high sensitivity and a negative predictive value, especially for patients with moderate to severe OSA. ${ }^{(6)}$

Recent studies have demonstrated the utility of the STOPBANG questionnaire in stratifying patients undergoing elective surgery. ${ }^{(1,7)}$ Vasu et al showed that patients categorised as having a high risk of OSA using the STOP-BANG questionnaire (i.e. had scores $\geq 3$ ) were associated with a higher rate of postoperative complications as compared to patients with STOP-BANG scores $<3(19.6 \%$ vs. $1.3 \%, \mathrm{p}<0.001) .{ }^{(1)}$ Chia et al showed that higher preoperative STOP-BANG scores were significantly associated with postoperative critical care admission. ${ }^{(7)}$

In the present study, we hypothesised that elective surgical patients with higher preoperative STOP-BANG scores are associated with an increased likelihood of intraoperative and early postoperative adverse outcomes. We explored the novel use of STOP-BANG scores for preoperative risk stratification of adverse events for elective surgical patients.

\section{METHODS}

This retrospective cohort study was approved by the Domain Specific Review Board and funded by the Alexandra Health Enabling Grant 2011. Preoperative assessment of the patients who 
Table I. STOP-BANG questionnaire for screening of obstructive sleep apnoea.

\begin{tabular}{ll}
\hline Item & Question \\
\hline 1. Snoring & $\begin{array}{l}\text { Do you snore loudly (louder than } \\
\text { talking or loud enough to be heard } \\
\text { through closed doors)? }\end{array}$ \\
2. Tired & $\begin{array}{l}\text { Do you often feel tired, fatigued or } \\
\text { sleepy during the daytime? }\end{array}$ \\
3. Observed & Has anyone observed you stop \\
& breathing during your sleep? \\
4. Blood Pressure & Are you being, or have been, treated \\
& for high blood pressure? \\
5. Body mass index & Is your body mass index $>35 \mathrm{~kg} / \mathrm{m}^{2} ?$ \\
6. Age & Are you $>50$ years old? \\
7. Neck circumference & Is your neck circumference $>40 \mathrm{~cm} ?$ \\
8. Gender & Are you male?
\end{tabular}

underwent elective surgery was performed in the preoperative anaesthesia clinic of Khoo Teck Puat Hospital, Alexandra Health Services, Singapore, a tertiary teaching hospital. The preoperative assessment was conducted over a one-year period, from 1 January 2011 to 31 December 2011. Patients who underwent elective ophthalmic surgeries or emergency surgeries were excluded from this study.

Information obtained from the preoperative assessment of elective surgical patients was recorded and digitised into the OTSystem (Medisys Innovation Pte Ltd, Singapore), the hospital's proprietary, customised perioperative electronic medical record system. Mandatory information collected in the perioperative records included demographic data (i.e. age, gender and ethnicity), clinical data (i.e. American Society of Anesthesiologists [ASA] physical status, comorbidities, body mass index [BMI] and STOP-BANG score), surgical data (i.e. nature of surgery and type of anaesthesia used) and unexpected perioperative adverse events as reported by the attending anaesthetist. The perioperative adverse events recorded (detailed in Appendix) were mainly intraoperative and early postoperative events, as they were defined as such in the hospital's medical record system. In the present study, there were no cases of perioperative adverse events unrelated to OSA reported (e.g. 'nerve injury', 'adverse drug reaction' and 'equipment failure'). The primary outcome of this study was the occurrence of unexpected intraoperative and early postoperative adverse events. The patients' demographic data, STOP-BANG score, ASA physical status, comorbidities and surgical data were evaluated as predictors of the aforementioned outcome.

Data was analysed using the IBM Statistical Package for the Social Sciences software for Windows version 20.0 (IBM Corp, Armonk, NY, USA). Continuous variables were reported as mean \pm standard deviation, while categorical variables were reported as number (percentage). Multiple logistic regression analysis was used to assess the effect of individual risk factors on unexpected perioperative adverse events, after adjustment for other factors. Odds ratios (ORs) and their corresponding 95\% confidence intervals (Cls) were reported. A p-value $<0.05$ was considered statistically significant.
Table II. Demographic data of the patients $(n=5,432)$.

\begin{tabular}{|c|c|}
\hline Variable & No. (\%) \\
\hline \multicolumn{2}{|l|}{ Age (yr) } \\
\hline$>60$ & $1,055(19.4)$ \\
\hline$\leq 60$ & $4,377(80.6)$ \\
\hline \multicolumn{2}{|l|}{ Gender } \\
\hline Male & $3,578(65.9)$ \\
\hline Female & $1,854(34.1)$ \\
\hline \multicolumn{2}{|l|}{ Ethnicity } \\
\hline Chinese & $3,835(70.6)$ \\
\hline Malay & $801(14.7)$ \\
\hline Indian & $480(8.8)$ \\
\hline Others & $316(5.8)$ \\
\hline Obese $\left(B M I ~>30 \mathrm{~kg} / \mathrm{m}^{2}\right)^{*}$ & $565(11.4)$ \\
\hline \multicolumn{2}{|l|}{ ASA class* } \\
\hline 1 & $2,328(44.5)$ \\
\hline 2 & $2,328(44.5)$ \\
\hline 3 & $535(10.2)$ \\
\hline 4 & $42(0.8)$ \\
\hline Known obstructive sleep apnoea & $117(2.2)$ \\
\hline \multicolumn{2}{|l|}{ Comorbidities } \\
\hline Chronic obstructive pulmonary disease & $22(0.4)$ \\
\hline Hypertension (controlled) & $1,161(21.4)$ \\
\hline Hypertension (uncontrolled) & $42(0.8)$ \\
\hline Diabetes mellitus & $447(8.2)$ \\
\hline Hypothyroidism & $42(0.8)$ \\
\hline Hyperthyroidism & $37(0.7)$ \\
\hline Cerebrovascular accident & $113(2.1)$ \\
\hline Asthma & $457(8.4)$ \\
\hline Smoking & $1,587(29.2)$ \\
\hline \multicolumn{2}{|l|}{ Type of surgery } \\
\hline Ear, nose and throat & $486(8.9)$ \\
\hline General & $2,068(38.1)$ \\
\hline Oral maxillofacial & $1,622(29.9)$ \\
\hline Orthopaedic & $1,256(23.1)$ \\
\hline
\end{tabular}

* BMI data missing for 473 patients and ASA class missing for 199 patients. ASA: American Society of Anaesthesiologists; BMI: body mass index

\section{RESULTS}

A total of 9,178 patients were administered the STOP-BANG questionnaire during their review in the preoperative anaesthesia clinic. Among these patients, 3,746 patients were excluded from the present study as they underwent either ophthalmic surgery or emergency surgery. Thus, a total of 5,432 patients were included in the study.

The baseline demographic data of the 5,432 patients is shown in Table II. The mean patient age was $40.8 \pm 19.1$ years; $19.4 \%$ of the patients were aged $>60$ years. Most of the patients were male $(65.9 \%) ; 34.1 \%$ were female. Among the patients, $11.4 \%$ had a BMI $>30 \mathrm{~kg} / \mathrm{m}^{2}$. Only $2.2 \%$ of the patients had a previous diagnosis of OSA. High-risk patients (i.e. ASA class 3 and 4 ) comprised $11.0 \%$ of all the patients. The most common type of surgery was general surgery $(38.1 \%)$, followed by oral maxillofacial surgery (29.9\%), orthopaedic surgery $(23.1 \%)$ and ear, nose and throat surgery $(8.9 \%)$. Our hospital does not perform 
cardiac, and obstetrics and gynaecological surgeries. During the study period, neurosurgical procedures were performed on an emergency-only basis and these were excluded from our study.

Of the 5,432 patients, $485(8.9 \%)$ patients had STOP-BANG scores of $3-8$, and 76 (1.4\%) patients had STOP-BANG scores of $5-8 ; 7.4 \%$ had unexpected intraoperative and early postoperative adverse events. Patients with higher STOP-BANG scores were found to have a higher risk of unexpected intraoperative and early postoperative adverse events (Table III). The ORs of unexpected intraoperative and early postoperative adverse events for patients with STOP-BANG scores of 3, 4, 5 and $\geq 6$ vs. 0 were $3.6(95 \%$ Cl 2.1-6.3), 3.4 (95\% Cl 1.8-6.5), 6.4 (95\% Cl 2.7-15.0) and 5.6 (95\% Cl 2.1-15.4), respectively. When stratified according to STOP-BANG scores, the risk of having an unexpected intraoperative and early postoperative adverse event was found to rise with increasing STOP-BANG scores (Table IV). Patients with STOP-BANG scores $<3$ had a $5.5 \%$ chance of having an adverse event, while patients with STOP-BANG scores of 3-4, $5-6$ and $7-8$ had a $25.4 \%, 34.3 \%$ and $44.4 \%$ chance of having an adverse event, respectively. From the correlates included in the multiple logistic regression model, other independent predictors of unexpected intraoperative and early postoperative adverse events were older age $(p<0.001)$, ASA class $\geq 2(p<0.003)$ and uncontrolled hypertension ( $p=0.028)$ (Table III).

\section{DISCUSSION}

OSA is a chronic multisystem disorder that has been associated with cardiovascular diseases (e.g. ischaemic heart disease, heart failure, arrhythmias and hypertension), cerebrovascular diseases (stroke and transient ischaemic attack) and endocrine disorders (e.g. metabolic syndrome and obesity). ${ }^{(8)}$ These comorbidities, together with the pathophysiological effects of sedatives and/or analgesic and anaesthetic agents, may aggravate the symptoms of OSA by reducing pharyngeal tone, ventilatory reflexes and arousal responses, consequently leading to airway obstruction, hypoxia, hypercarbia, haemodynamic aberrations and other adverse events during the perioperative period. ${ }^{(9)} \mathrm{A}$ meta-analysis by Kaw et al showed that OSA increased the odds of postoperative complications such as myocardial infarction, cardiac arrest, arrhythmias, respiratory failure, desaturation, intensive care unit transfers and reintubations. ${ }^{(3)}$

Higher STOP-BANG scores were shown to be correlated to higher rates of postoperative respiratory and cardiovascular complications in a study conducted by Vasu et al. ${ }^{(1)}$ In the present study, we investigated the relationship between the rate of unexpected intraoperative and early postoperative complications (not limited to respiratory and cardiovascular complications) and STOP-BANG scores. We found that STOPBANG scores were useful for stratifying patients who are at risk of unexpected intraoperative and early postoperative adverse events. Patients with higher STOP-BANG scores (i.e. $\geq 3$ ) were found to have higher risks of perioperative adverse events. The ORs of unexpected adverse events for patients with STOP-BANG scores of 3 and 4 (vs. 0) were 3.6 and 3.4, respectively. STOPBANG scores of 5 and $\geq 6$ were associated with at least a fivefold
Table III. Risk estimates for unexpected perioperative adverse events.

\begin{tabular}{llc}
\hline Variable & OR (95\% CI) & $\begin{array}{c}\text { Adjusted } \\
\text { p-value }\end{array}$ \\
\hline Age $^{\dagger}$ & $1.023(1.013-1.033)$ & $<0.001^{*}$ \\
STOP-BANG score $^{*}$ & $1.2(0.8-1.9)$ & 0.365 \\
1 & $1.4(0.8-2.2)$ & 0.236 \\
2 & $3.6(2.1-6.3)$ & $<0.001^{*}$ \\
3 & $3.4(1.8-6.5)$ & $<0.001^{*}$ \\
4 & $6.4(2.7-15.0)$ & $<0.001^{*}$ \\
5 & $5.6(2.1-15.4)$ & $<0.001^{*}$ \\
$\geq 6$ & $1.8(1.3-2.6)$ & $<0.001^{*}$ \\
ASA class & & $0.003^{*}$ \\
2 & $2.1(1.3-3.4)$ & $0.003^{*}$ \\
3 & $3.2(1.5-6.8)$ & 0.58 \\
$\geq 4$ & $1.2(0.7-2.1)$ & 0.59 \\
History of OSA & $1.1(0.7-1.6)$ & $0.028^{*}$ \\
Asthma & $2.4(1.1-5.0)$ & \\
Uncontrolled hypertension & &
\end{tabular}

$*_{p}<0.05$ is considered statistically significant. ${ }^{\dagger}$ Age was analysed as a continuous variable; an increase in 1 year increases the odds of perioperative adverse event by 2.3\%. \#STOP-BANG score analysed in comparison to STOP-BANG score 0. "ASA class analysed in comparison to ASA class 1. ASA: American Society of Anesthesiologists; $\mathrm{Cl}$ : confidence interval; OR: odds ratio; OSA: obstructive sleep apnoea

Table IV. Number of patients with perioperative adverse events according to stratified STOP-BANG scores.

\begin{tabular}{lcc}
\hline $\begin{array}{l}\text { STOP-BANG } \\
\text { score }\end{array}$ & $\begin{array}{c}\text { No. of } \\
\text { patients }\end{array}$ & $\begin{array}{c}\text { No. (\%) of patients } \\
\text { with adverse events }\end{array}$ \\
\hline 0 & 1,069 & $29(2.7)$ \\
$1-2$ & 3,878 & $243(6.3)$ \\
$3-4$ & 409 & $104(25.4)$ \\
$5-6$ & 67 & $23(34.3)$ \\
$7-8$ & 9 & $4(44.4)$ \\
\hline
\end{tabular}

increased rate of perioperative adverse events (OR 6.4, 95\% CI 2.7-15.0, $\mathrm{p}<0.001$ and OR 5.6, 95\% Cl 2.1-15.4, $\mathrm{p}<0.001$, respectively). As the number of patients with STOP-BANG scores $\geq 6$ was small, this could have contributed to the lower OR observed for these patients as compared to that of the patients with a STOP-BANG score of 5.

In the present study, stratification of the STOP-BANG scores also showed that increasing scores were associated with an increasing risk of unexpected intraoperative and early postoperative adverse events. This means that the STOP-BANG score may be ideal for the stratification of intraoperative and early postoperative risk. In our study, patients with STOP-BANG scores $\geq 3$ were shown to have at least a 'one in four' chance of an adverse event in the intraoperative and early postoperative period. Approximately $44.4 \%$ of the patients with STOP-BANG scores of 7 and 8 were at risk of an adverse event. This finding is in keeping with the results of a smaller sample size study by Vasu et al. ${ }^{(1)}$ In that study, 135 mixed surgical patients who had STOP-BANG scores $\geq 3$ and ASA class $\geq 3$, were found to have a higher risk of postoperative pulmonary or cardiac complications, and an increased length of hospital stay. ${ }^{(1)} \mathrm{A}$ recent study by Chia et al also 
showed that a STOP-BANG score $\geq 4$ was significantly associated with an increased risk of critical care admission. ${ }^{(7)}$

Interestingly, we did not find a correlation between patients known to have OSA and perioperative adverse events in our study. However, the number of patients with known OSA in this study was small and this may have led to the inability to detect a statistically significant correlation. As pointed out by Memtsoudis et al, about four out of five patients presenting for surgery have undetected OSA. ${ }^{(2)}$ Other than that, patients with known or diagnosed OSA may be on treatment and continuous positive airway pressure therapy, thus reducing their perioperative risks.

Previous studies have described other risk factors associated with perioperative complications, including advanced age, ASA class $\geq 3$, congestive heart failure, obesity and hypoalbuminaemia. ${ }^{(1,10)}$ Some of these independent predictors of unexpected perioperative adverse events were also detected in the present study, namely older age $(p<0.001)$, ASA class $\geq 2$ $(p<0.003)$ and uncontrolled hypertension $(p=0.028)$.

OSA may be incorrectly diagnosed if the diagnosis is based solely on the STOP-BANG questionnaire; this is because the STOP-BANG questionnaire has low specificity. ${ }^{(11)}$ In the present study, the diagnosis of OSA was not confirmed with polysomnography for the patients who had high STOP-BANG scores, as this was not part of the study protocol. In view of the evidence that suggests a correlation between OSA and increased perioperative adverse events, it may be prudent for patients with STOP-BANG scores $\geq 5$ to undergo polysomnography prior to elective surgery. Risk mitigation strategies would be important in these high-risk patient groups.

The present study was not without limitations. It was limited by the attending anaesthetist's reporting of information (e.g. comorbidities, STOP-BANG score and perioperative adverse events) on the OTSystem. Also, as the study was done retrospectively, we were not able to account for missing data or include other data that may be of importance (e.g. the timing of the perioperative adverse events and/or specific data regarding cardiovascular or respiratory adverse events).

In conclusion, the present study demonstrated that akin to the ASA physical status classification, the STOP-BANG score may be used as a novel preoperative risk-stratification tool. A STOP-BANG score $\geq 5$ was found to be associated with a fivefold increased risk of unexpected intraoperative and early postoperative events. One in four patients with STOP-BANG scores $\geq 3$ was found to be at risk of an adverse event. Therefore, attending anaesthetists should be vigilant in the management of these at-risk patients.

\section{ACKNOWLEDGEMENTS}

We thank A/Prof Ng Tze Pin from Yong Loo Lin School of Medicine, National University of Singapore, Singapore, for his assistance with the statistical analyses.

\section{REFERENCES}

1. Vasu TS, Doghramji K, Cavallazzi R, et al. Obstructive sleep apnea syndrome and postoperative complications: clinical use of the STOPBANG questionnaire. Arch Otolaryngol Head Neck Surg 2010; 136:1020-4

2. Memtsoudis SG, Besculides MC, Mazumdar M. A rude awakening--the perioperative sleep apnea epidemic. N Engl J Med 2013; 368:2352-3

3. Kaw R, Chung F, Pasupuleti V, et al. Meta-analysis of the association between obstructive sleep apnoea and postoperative outcome. Br J Anaesth 2012; 109:897-906.

4. Mokhlesi B, Hovda MD, Vekhter B, et al. Sleep-disordered breathing and postoperative outcomes after elective surgery: analysis of the nationwide inpatient sample. Chest 2013; 144:903-14.

5. Chung F, Yegneswaran B, Liao P, et al. STOP questionnaire: a tool to screen patients for obstructive sleep apnea. Anesthesiology 2008; 108: 812-21.

6. Chung F, Subramanyam R, Liao P, et al. High STOP-Bang score indicates a high probability of obstructive sleep apnoea. Br J Anaesth 2012; 108:768-75

7. Chia P, Seet E, Macachor JD, Iyer US, Wu D. The association of preoperative STOP-BANG scores with postoperative critical care admission. Anaesthesia 2013; 68:950-2

8. Seet E, Chung F. Obstructive sleep apnea: preoperative assessment. Anesthesiol Clin 2010; 28:199-215.

9. Ankichetty S, Wong J, Chung F. A systematic review of the effects of sedatives and anesthetics in patients with obstructive sleep apnea. J Anaesthesiol Clin Pharmacol 2011; 27:447-58.

10. Qaseem A, Snow V, Fitterman N, et al; Clinical Efficacy Assessment Subcommittee of the American College of Physicians. Risk assessment for and strategies to reduce perioperative pulmonary complications for patients undergoing noncardiothoracic surgery: a guideline from the American College of Physicians. Ann Intern Med 2006; 144:575-80.

11. Seet E, Chung F. Management of sleep apnea in adults - functional algorithms for the perioperative period: Continuing Professional Development. Can J Anaesth 2010; 57:849-64. 


\section{APPENDIX}

\section{Definitions of unexpected perioperative events as captured by the medical record system.}

1. Hypoxia

2. Failed intubation

3. Dental injury

4. Laryngospasm

5. Bronchospasm

6. Arrhythmia

7. Hypertension

8. Hypotension

9. Haemorrhage

10. Hypothermia

11. Nerve injury

12. Drug adverse reaction

13. Equipment failure

14. Unplanned high dependency unit (HDU) admit

15. Unplanned intensive care unit (ICU) admit 16. Post-anaesthesia care unit (PACU) $>2$ hours
Clinically significant oxygen desaturation to $<90 \%$ requiring change in management

Multiple attempts and eventual failure in the placement of a tracheal tube into the trachea

Injuries to the patient within 7 days of their anaesthetics that required dental consultation and led to a recommendation to repair, stabilise, or extract involved dentition or supporting structures

Airway obstruction due to uncontrolled muscular contraction of laryngeal cords requiring treatment

Clinically significant wheezing and decrease airway entry during anaesthesia due to bronchial smooth muscle contraction requiring treatment

New or worsening disturbance of heart rhythm requiring new treatment or a change in treatment

Increase in systolic pressure to $>200 \mathrm{mmHg}$ or diastolic pressure to $>110 \mathrm{mmHg}$ with new antihypertensive or change in treatment required

Decrease in systolic pressure $<90 \mathrm{mmHg}$ with treatment required Unanticipated bleeding from the surgery requiring blood transfusion

Core body temperature (oesophagus, tympanic membrane) verified to be $<35$ degrees Celsius

Clinically significant motor and/or sensory impairment after general anaesthesia, peripheral nerve block, or neuroaxial block

Harm associated with the use of given medication at a normal dose

Failure of medical equipment related to the conduct of anaesthesia potentially resulting or actually resulting in patient harm

Unanticipated HDU admission for medical, surgical or anaesthetic reasons

Unanticipated ICU admission for medical, surgical or anaesthetic reasons

a. Postoperative nausea and vomiting (PONV) - PACU stay for longer than 2 hours due to severe PONV necessitating treatment

b. Pain - PACU stay for longer than 2 hours due to severe pain or visual analogue pain score $>7 / 10$

c. Sedation - PACU stay for longer than 2 hours due to patient sedation (drowsy and difficult to rouse) 